\title{
A SUFFICIENT CONDITION FOR AN EXTREME COVERING OF n-SPAGE BY SPHERES
}

\author{
T. J. DICKSON
}

(Received 29 August 1966)

\section{1}

The problem of finding the most economical coverings of $n$-dimensional Euclidean space by equal spheres whose centres form a lattice, which is equivalent to a problem concerning the inhomogeneous minima of positive definite quadratic forms, has been discussed recently by Barnes and Dickson [1]. The reader is referred to [1] for a complete background on the problem. Terms and notations used will be as in that paper.

Among other results, Barnes and Dickson proved that if $f$ is an extreme form in the interior of a Voronoï cone $\Delta$ then (i) every extreme form in $\Delta$ is a multiple of $f$ and (ii) $f$ and $\Delta$ have the same group of automorphisms. It is the main object of this paper to extend these results to the following necessary and sufficient condition for extremeness.

THEOREM. If $\mathscr{F}$ is the set of all forms in a Voronoi cone $\Delta$ which are invariant under all automorphisms of $\Delta$, and $f$ is a form in the interior of a Voronoi cone $\Delta$ then $f$ is extreme if and only if $f \in \mathscr{F}$ and

$$
\mu(f)=\min _{\boldsymbol{\sigma} \in \mathscr{F}} \mu(g)
$$

$\S \S 2$ and 3 are concerned with proving this result and $\S 4$ with applying it to the known Voronoî cones.

In $\S 5$ the case where the only form satisfying (1.1) lies on the boundary of the cone is discussed.

\section{2}

The following two lemmas are necessary for the proof of the above theorem.

LEMMA 1. If $f$ is an interior form, $\varphi$ any quadratic form, then the inequality

$$
\mu(f+\varepsilon \varphi) \leqq \mu(f)
$$

holds for all sufficiently small $\varepsilon$ (of either sign) if and only if $\phi$ is a multiple of $t$. 
This result appeared in [1] but was submerged in the proof of Theorem 2 of that paper. It was not stated as a separate result at that stage.

This lemma means that in the interior of a Voronoi cone the function $\mu(f)$ cannot have a local maximum on a line i.e. the maximum of $\mu(f)$ on a line segment must occur at one or both of the end points.

LEMMA 2. If $k$ forms $f_{0}, f_{1}, \cdots, f_{k-1}$ are in the interior of the same Voronoi cone and are not all multiples of the same form, then

$$
\mu\left(f_{0}+f_{1}+\cdots+f_{k-1}\right)<\max _{i} \mu\left(f_{i}\right) .
$$

Proof. The lemma will be proved by induction on $k$.

(i) $k=2$.

Consider the forms

$$
f_{t}(x)=(1-t) f_{0}(x)+t f_{1}(x)
$$

for $0 \leqq t \leqq 1$. If the maximum value of $\mu\left(f_{t}\right)$ is attained at a point, $f_{t_{1}}$ say, for which $0<t_{1}<1$, then

$$
\mu\left(f_{t_{1}}+\varepsilon\left(f_{0}-f_{1}\right)\right) \leqq \mu\left(f_{t_{1}}\right)
$$

for all sufficiently small $\varepsilon$ of either sign.

This is impossible, by Lemma 1 , as $t_{0}$ is not a multiple of $f_{1}$ and so $\mu\left(f_{t}\right)<\max _{i=0,1} \mu\left(f_{i}\right)$ for all $0<t<1$.

Taking $t=\frac{1}{2}$ and remembering that $\mu(\lambda f)=\mu(f)(\lambda>0)$, we have

$$
\mu\left(f_{0}+f_{1}\right)<\max _{i=0,1} \mu\left(f_{i}\right)
$$

(ii) Assume (2.1) true for $k \leqq K$ and consider case $k=K+1$.

Without loss of generality we can assume that $f_{0}, f_{1}, \cdots, f_{K-1}$ are not all multiplies of the same form and $f_{K}$ is not a multiple of $f_{0}+f_{1}+\cdots+f_{K-1}$.

By assumption

and, from (2.2),

$$
\mu\left(f_{0}+f_{1}+\cdots+f_{K-1}\right)<\max _{i=0, \cdots, K-1} \mu\left(f_{i}\right)
$$

$$
\mu\left(t_{0}+\cdots+f_{k}\right)<\max \left\{\mu\left(f_{K}\right), \mu\left(f_{0}+\cdots+f_{K-1}\right)\right\} .
$$

(2.3) and (2.4) together give (2.1) and so (2.1) holds for all $k$.

\section{Proof of the Theorem}

(i) Let $f$ be an extreme interior form of a Voronoï cone $\Delta$.

From the results of Barnes and Dickson [1], $f \in \mathscr{F}$ and obviously

$$
\mu(f) \leqq \mu(g)
$$

for all $g \in \mathscr{F}$ sufficiently close to $f$. 
Let $f_{0}$ be a form in $\mathscr{F}$ such that $\mu\left(f_{0}\right)=\min _{g \in \mathscr{F}} \mu(g)$ and consider line segment of forms

$$
f_{t}(x)=t f(x)+(1-t) f_{0}(x)
$$

for $0 \leqq t \leqq 1$.

By (3.1) and the definition of $f_{0}$, the maximum value of $\mu\left(f_{t}\right)$ is attained at a point $f_{t_{1}}$ for which $0<t_{1}<1$ and so

$$
\mu\left(f_{t_{1}}+\varepsilon\left(t_{0}-f\right)\right) \leqq \mu\left(f_{t_{1}}\right)
$$

for all sutficiently small $\varepsilon$ of either sign. By lemma $1, f_{0}-f$ is a multiple of $t_{t_{1}}$ i.e. $t_{0}$ is a multiple of $t$.

Thus $\mu(f)=\min _{g \in \mathscr{F}} \mu(g)$.

(ii) Let $f$ be an interior form of $\Delta$ such that $f \in \mathscr{F}$ and

$$
\mu(f)=\min _{g \in \mathscr{F}} \mu(g) .
$$

Let there be another form $f_{0}$ in $\Delta$ for which $\mu\left(f_{0}\right)<\mu(f)$.

Obviously $f_{0} \notin \mathscr{F}$.

Consider the forms obtained by applying all automorphisms of $\Delta$ to $f_{0}$ and let these forms be $f_{i}(i=0, \cdots, k-1)$.

As $f_{0} \notin \mathscr{F}$, not all the $f_{i}$ are multiples of the same form.

Now $\mu\left(f_{i}\right)=\mu\left(f_{0}\right)$ for $(i=0, \cdots, k-1)$, so, by Lemma 2

$$
\mu\left(f_{0}+f_{1}+\cdots+f_{k-1}\right)<\mu\left(f_{0}\right)<\mu(f) .
$$

But $f_{0}+f_{1}+\cdots+f_{k-1} \in \mathscr{F}$ and we have a contradiction to (3.2).

Thus $\mu(g) \geqq \mu(f)$ for all $g$ in $\Delta$ and hence $f$ is extreme.

The proof is now complete.

In (i) of the above proof, if $f_{0}$ had been defined as any form for which

$$
\mu\left(f_{0}\right)=\min _{g \in \Delta} \mu(g)
$$

then the same argument would give that $f_{0}$ is a multiple of $f$. Thus we have:

COROLLARY. If $f$ is an extreme interior form of $\Delta$ then

$$
\mu(g)>\mu(f)
$$

for all $g$ in $\Delta$ not a multiple of $f$.

\section{Applications}

Dickson extracted all the extreme quaternary forms [2] by finding the set $\mathscr{F}$ for each cone and minimizing $\mu(f)$ over $\mathscr{F}$ in each case, i.e. finding the forms satisfying (1.1). However, as this condition had not been shown to be sufficient for extremeness, another result of $[1]$ had to be used 
to prove that the forms were in fact extreme and the uniqueness of the extreme form in any Voronoi cone used to conclude that they were the only extreme forms. With the above theorem, however, we see that we can conclude immediately that the respective forms are extreme. A similar comment applies to the treatment of the principal domain in [1] by Barnes and Dickson.

The following discussion of the known Voronoi cones shows how easily the extreme forms can be extracted using the above theorem.

(i) The principal domain

For every $n \geqq 2$, the principal domain $\Delta$ is the set of forms expressible in the form

$$
\phi(x)=\sum_{\substack{i, j=0 \\ i<j}}^{n} \rho_{i j}\left(x_{i}-x_{j}\right)^{2}, \quad x_{0}=0, \quad \rho_{i j} \geqq 0 \quad(0 \leqq i<j \leqq n) .
$$

The group $G(\Delta)$ of automorphisms of $\Delta$ has order $2(n+1)$ ! and is transitive on the edge forms $\left(x_{i}-x_{j}\right)^{2}(0 \leqq i<j \leqq n)$ of $\Delta . G(\Delta)$ is in fact generated by (i) all permutations of $x_{1}, x_{2}, \cdots, x_{n}$; (ii) $x_{i} \rightarrow-x_{i}(1 \leqq i \leqq n)$; (iii) $x_{1} \rightarrow x_{1}, x_{i} \rightarrow x_{1}-x_{i}(2 \leqq i \leqq n)$.

So the set $\mathscr{F}$ as defined in the above theorem contains only multiples of

$$
f(x)=\sum_{\substack{i, j=0 \\ i<j}}^{n}\left(x_{i}-x_{j}\right)^{2}=n \sum_{i=1}^{n} x_{i}^{2}-2 \sum_{1 \leqq i<j \leqq n} x_{i} x_{i} .
$$

As this is an interior form then by the above theorem it is extreme and there are no other extreme forms (including boundary forms) in $\Delta$.

(ii) The cone $\Delta^{\prime \prime}$ of quaternary forms

$\Delta^{\prime \prime}$ is the set of forms expressible in the form

$$
\begin{aligned}
\phi(x)= & \rho_{1} x_{1}^{2}+\rho_{2} x_{2}^{2}+\rho_{3} x_{3}^{2}+\rho_{4} x_{4}^{2}+\rho_{12} \omega+\rho_{13}\left(x_{1}-x_{3}\right)^{2} \\
& +\rho_{14}\left(x_{1}-x_{4}\right)^{2}+\rho_{23}\left(x_{2}-x_{3}\right)^{2}+\rho_{24}\left(x_{2}-x_{4}\right)^{2}+\rho_{34}\left(x_{1}+x_{2}-x_{3}-x_{4}\right)^{2}
\end{aligned}
$$

where $\rho_{i} \geqq 0, \rho_{i j} \geqq 0$ for all $i, j$ and

$$
\omega=2 x_{1}^{2}+2 x_{2}^{2}+2 x_{3}^{2}+2 x_{4}^{2}+2 x_{1} x_{2}-2 x_{1} x_{3}-2 x_{1} x_{4}-2 x_{2} x_{3}-2 x_{2} x_{4} .
$$

The group $G\left(\Delta^{\prime \prime}\right)$ of automorphisms of $\Delta^{\prime \prime}$ contains the transformations (i) $x_{1} \leftrightarrow x_{2}$ (ii) $x_{3} \leftrightarrow x_{4}$ (iii) $x_{1} \rightarrow x_{1}-x_{3}, x_{2} \rightarrow x_{4}, x_{3} \rightarrow x_{1}, x_{4} \rightarrow x_{4}-x_{2}$. The family of forms of $\Delta^{\prime \prime}$ (neglecting boundary forms) which are invariant under these transformations are those expressible in the form

$$
\begin{aligned}
f(x)= & x_{1}^{2}+x_{2}^{2}+x_{3}^{2}+x_{4}^{2}+\left(x_{1}-x_{3}\right)^{2}+\left(x_{1}-x_{4}\right)^{2}+\left(x_{2}-x_{3}\right)^{2}+\left(x_{2}-x_{4}\right)^{2} \\
& +\left(x_{1}+x_{2}-x_{3}-x_{4}\right)^{2}+\alpha \omega(\alpha>0) .
\end{aligned}
$$


As $\omega$ is not equivalent to the other edge forms of $\Delta^{\prime \prime}$, then this is the set $\mathscr{F}$ (neglecting boundary forms).

By using Voronoï's table of vertices for the cone $\Delta^{\prime \prime}$ [3p. 173], as in [2], we obtain that

for all $f$ in $\mathscr{F}$.

$$
\mu(f)=\frac{2\left(2-3 \alpha+3 \alpha^{2}-\alpha^{3}\right)}{(1+\alpha)^{\frac{3}{2}}(2-\alpha)^{\frac{1}{2}}}
$$

Solving $\partial \mu(f) / \partial \alpha=0$, we obtain $\alpha=\frac{1}{2}(5-\sqrt{ } 13)$.

As this value of $\alpha$ gives an interior form of $\Delta^{\prime \prime}$ then $f(x)$ is extreme when $\alpha=\frac{1}{2}(5-\sqrt{ } 13)$ and this is the only extreme form in $\Delta^{\prime \prime}$ (neglecting multiples).

(iii) The cone $\Delta^{\prime}$.

In a similar manner the form $f_{3}(x)$ of $[2]$ can be found to be the extreme form in the cone $\Delta^{\prime}$ of quaternary forms. Consider, however, the general cone $\Delta^{\prime}[3$, p. 159].

For all $n \geqq 4$, the cone $\Delta^{\prime}$ is the set of forms expressible in the form

$$
\phi(x)=\sum_{i=1}^{n} \rho_{i} x_{i}^{2}+\sum_{\substack{i<j \\ i \neq \neq 12}} \rho_{i j}\left(x_{i}-x_{j}\right)^{2}+\rho_{12} \omega,
$$

where $\rho_{i} \geqq 0, \rho_{i j} \geqq 0$ for all $i, j$ and

$$
\omega=(n-2)\left(x_{1}^{2}+x_{2}^{2}\right)+2 \sum_{i=3}^{n} x_{i}^{2}+2 x_{1} x_{2}-2 \sum_{i=3}^{n}\left(x_{1}+x_{2}\right) x_{i} .
$$

It can be shown rather laboriously, making use once again of the above theorem, that an interior form in this cone is extreme if and only if it is a multiple of

$$
\begin{aligned}
f(x)= & (n-1-\alpha)\left(x_{1}^{2}+x_{2}^{2}\right)+(2+(n-2) \beta) \sum_{i=3}^{n} x_{i}^{2}+2 \alpha x_{1} x_{2} \\
& -2 \sum_{i=3}^{n}\left(x_{1}+x_{2}\right) x_{i}-2 \beta \sum_{i<j} x_{i} x_{j},
\end{aligned}
$$

where $\alpha, \beta$ satisfy

$$
\begin{aligned}
& \left\{\begin{array}{l}
0<\alpha<1, \\
\beta>0, \\
n-2-2 \alpha-\alpha \beta>0,
\end{array}\right. \\
& 6(\beta+2)(1-\alpha)\left\{\left(n^{2}-n-1\right)+(1-2 n) \alpha\right\} \\
& =n(n-1)(n-2) \beta^{2}+6 n(n-2) \beta+12(n-2) \text {, } \\
& 3(1-\alpha)^{2}\{(n-1) \beta+2(2 n-1)\} \\
& =n(n-1)^{2} \beta^{2}-n(n-1)(n-6) \beta-6 n^{2}+24 n-12 \text {. }
\end{aligned}
$$




\section{Boundary forms}

All the extreme forms found so far are interior forms, so there has been no results yet which disprove the conjecture made previously that all extreme forms are interior forms.

For each Voronoi cone, the set $\mathscr{F}$ is non-empty and closed (the form $\sum_{i} \phi_{i}(x)$ where $\phi_{i}(x)(i=1, \cdots, N)$ are all the edge forms of the cone, must belong to $\mathscr{F})$. Thus there will be at least one form $f_{0}$ in $\mathscr{F}$ for which

$$
\mu\left(f_{0}\right)=\min _{\boldsymbol{g} \in \mathscr{F}} \mu(g) .
$$

We already know that if there is an interior form $t_{0}$ in $\mathscr{F}$ satisfying (5.1) then (neglecting multiples of $f_{0}$ ),

(i) $f_{0}$ is the only form in $\mathscr{F}$ satisfying (5.1)

(ii) $t_{0}$ is extreme and

(iii) $t_{0}$ is the only extreme form in $\Delta$, boundary forms included.

If, however, there is no interior form $f_{0}$ satisfying (5.1) i.e. the minimum of $\mu(f)$ on $\mathscr{F}$ is attained on the boundary then this implies that there are no extreme interior forms, but, as yet, it implies nothing about the existence or non-existence of extreme boundary forms.

Now, it can be shown that Lemma 1 can be extended to include the case where $f$ is a boundary form and $f+\varepsilon \varphi$ is also a boundary form for all sufficiently small $\varepsilon$. Using this the general theory can also be extended to include boundary forms. It is found that when there is a boundary form $f_{0}$ in $\mathscr{F}$ satisfying (5.1) then once again this is unique and also is still the only possible extreme form. However, in this case it is not necessarily extreme and will only be extreme if it satisfies (5.1) for all the cones in which it lies. We can summarize all cases as follows:

For any cone 4 , there will be a unique (up to multiplication by a constant) form $f_{0} \in \mathscr{F}$ satisfying (5.1) which is the only possible extreme form in $\Delta$. $t_{0}$ is extreme if and only if it is the unique form found in the same manner for all cones containing $f_{0}$.

The case where $f_{0}$ occurs on the boundary is definitely possible. The author has examined the general cone $\Delta^{\prime}$ for higher values of $n$ and found that there are no solutions of (4.2) and (4.3) which satisfy the conditions (4.1) for $n \geqq 14$. This means that there are no interior extreme forms in $\Delta^{\prime}$ for these values of $n$. The author has also found that for these values of $n$ the form $t_{0}$ as defined above lies on that boundary of $\Delta^{\prime}$ which is common with the principal domain. As the principal domain does not contain any boundary extreme forms then we know that $f_{0}$ is not extreme and hence $\Delta^{\prime}$ does not contain an extreme form, boundary or interior, for $n \geqq 14$.

Whether it is possible for $t_{0}$ to occur on the boundary of a cone and satisfy the above condition for extremeness, is still a matter for conjecture. 


\section{References}

[1] Barnes, E. S. and Dickson, T. J.: 'Extreme coverings of $n$-space by spheres', J. Aust. Math. Soc. 7 (1967), 115-127.

[2] Dickson T. J.: 'The extreme coverings of 4-space by spheres', J. Aust. Math. Soc. 7 (1967), 490-496.

[3] Voronoï, G.: 'Recherches sur les paralleloedres primitifs (Part 2) J. reine angew. Math. 136 (1909) 67-181.

The University of Western Australia 